\title{
Masificación universitaria y población estudiantil. El estudiantado de la UBA en dos décadas de democracia
}

\section{(1983-2004)}

\author{
Yann Cristal
}

\section{RESUMEN}

En el presente artículo se analizan las características sociodemográficas del estudiantado de la Universidad de Buenos Aires (UBA), Argentina y sus transformaciones en las primeras dos décadas de la actual democracia en Argentina, momento en que dicha institución triplicó su matrícula y se convirtió en una de las dos universidades con más alumnos de América Latina. A partir del análisis de censos universitarios y otros documentos, el texto se pregunta hasta qué punto la masificación universitaria supuso un proceso de democratización del acceso a la educación superior y cómo fue mutando la población estudiantil de la UBA en el periodo, caracterizada por una profunda heterogeneidad.

Palabras clave: Universidad de Buenos Aires, características del estudiante, características sociodemográficas, democracia, acceso a la educación superior, Argentina. 


\section{Massificação universitária e população estudantil. O estudantado da UBA em duas décadas de democracia (1983-2004)}

\section{RESUMO}

No presente artigo se analisam as características sociodemográficas do estudantado da "Universidad de Buenos Aires" (UBA), Argentina e suas transformações nas primeiras duas décadas da atual democracia na Argentina, momento em que dita instituição triplicou sua matrícula e se transformou em uma das duas universidades com mais alunos da América Latina. A partir da análise de censos universitários e outros documentos, o texto se pergunta até que ponto a massificação universitária implicou um processo de democratização do acesso à educação superior e como foi mudando a população estudantil da UBA no período, caracterizada por uma profunda heterogeneidade.

Palavras chave: Universidad de Buenos Aires, características do estudante, características sociodemográficas, democracia, acesso à educação superior, Argentina.

\section{University overcrowding and student population. The UBA student body in two decades of democracy (1983-2004)}

\section{ABSTRACT}

This article analyzes the sociodemographic characteristics of the student body at the University of Buenos Aires (UBA), Argentina, and its transformations during the first two decades of the current democracy in Argentina, when the institution tripled its enrollment and became one of the two universities with the most students in Latin America. Based on the analysis of university censuses and other documents, the text inquires to what extent university overcrowding meant a process of democratization of access to higher education and how the student population of the UBA changed during this period, which was characterized by a profound heterogeneity.

Key words: University of Buenos Aires, student characteristics, sociodemographic characteristics, democracy, access to higher education, Argentina. 


\section{Introducción}

Entre 1983 y 2004 la población estudiantil de la Universidad de Buenos Aires (UBA) prácticamente se triplicó, pasando de poco más de 100000 estudiantes a casi 300 000, número que tendió a mantenerse hasta la actualidad. ${ }^{1}$ Como señalan Buchbinder y Marquina (2008), este fuerte incremento de la matrícula fue una de las características más salientes de la universidad argentina en democracia. Si bien entre las décadas de 1950 y 1970 la UBA ya había asistido a un proceso de masificación universitaria, el salto que se produjo a partir de la salida de la última dictadura fue inédito. De este modo, la UBA es hoy una de las dos universidades con mayor cantidad de estudiantes de América Latina, junto a la Universidad Nacional Autónoma de México (UNAM).

Este fenómeno ocurrió en un país donde la población estudiantil constituye un porcentaje significativo de la juventud. Argentina cuenta con una tasa de estudiantes en la educación superior del 39\% de la población de edad de entre 20 y 24 años $^{2}$ y una tasa neta universitaria (estudiantes universitarios sobre población de edad) del 22\%. ${ }^{3}$ A la vez, el 78\% de esta población asiste a instituciones públicas, porcentaje entre los más altos de la región junto a Uruguay y Bolivia (Ferreyra et al., 2017). Estos rasgos dan cuenta de lo que Sandra Carli (2012:15) denomina la "tradición plebeya" de la universidad argentina, históricamente asociada a la incorporación de sectores medios a la formación universitaria. La base de esta masificación radica en el ingreso irrestricto y la gratuidad de la educación superior que distinguen a la universidad argentina de la mayoría de sus pares latinoamericanas. Sin embargo, la misma no está exenta de dificultades sustantivas en relación con la democratización en el acceso a la educación superior. Las altas tasas de deserción en el primer año y las bajas tasas de graduación que caracterizaron a la UBA durante el periodo, en el que también sufrió restricciones presupuestarias significativas, dan cuenta de problemáticas que no fueron suficientemente indagadas.

En función de estas cuestiones, en este trabajo buscamos aproximarnos a una respuesta a los siguientes interrogantes: ¿Hasta qué punto la masificación de la UBA en democracia supuso un acceso más amplio de sectores populares a la educación superior? ¿Cuáles fueron las características sociodemográficas de la población estudiantil desde 1983 y cómo fueron cambiando a lo largo de las dos décadas siguientes? ¿Se trata de una población estudiantil con rasgos relativamente similares o por el contrario profundamente heterogénea y con importantes diferencias en su seno?

Para investigar estas cuestiones nos apoyamos principalmente en el análisis de los cinco censos que realizó la Universidad de Buenos Aires en el periodo (1988, 1992, 1996, 2000, 2004) y en las series estadísticas publicadas por el Rectorado de la UBA. También contemplamos otras fuentes estadísticas como los anuarios de la Secretaría de Políticas Universitarias del Ministerio de Educación de la Nación e informes de la Organización para la Cooperación y el Desarrollo Económico (OCDE) y el Banco Mundial. Finalmente tomamos en cuenta trabajos como los de Sandra Carli (2012) y Mario Toer (1990 y 1998) a los que nos referiremos a continuación. ${ }^{4}$

\footnotetext{
${ }^{1}$ La UBA contaba en 2015 con 291744 alumnos (M de Ed. (2015), Anuario 2015, Estadísticas Universitarias Argentinas, Departamento de Información Universitaria, Secretaría de Políticas Universitarias, Ministerio de Educación de la Nación).

${ }^{2}$ Organización para la Cooperación y el Desarrollo Económico (OCDE), Education at a Glance 2017: OECD Indicators, DOI: http://dx.doi. org/10.1787/eag-2017-en.

${ }^{3}$ Anuario 2015, Estadísticas Universitarias Argentinas, Departamento de Información Universitaria, Secretaría de Políticas Universitarias, Ministerio de Educación de la Nación.

${ }^{4}$ Cabe aclarar que la investigación sobre la política estudiantil del periodo excede las posibilidades y objetivos de este trabajo. En este sentido, no se trata de un artículo sobre el movimiento estudiantil sino sobre las condiciones del estudiantado de la UBA en su conjunto. No obstante, este desarrollo podría contribuir a una mayor comprensión futura de los vínculos entre movimiento estudiantil y juventud universitaria en democracia.
} 


\section{Breve estado de la cuestión}

Las primeras investigaciones sobre la condición de los estudiantes universitarios argentinos, en particular de la Universidad de Buenos Aires, se remontan a las décadas de 1950 y 1960, coincidiendo con el primer proceso de masificación universitaria en el país y en momentos en los que la expansión de la educación superior suscitó creciente interés por parte de las ciencias sociales en distintas partes del mundo (el clásico libro de Bourdieu y Passeron (1964) es un ejemplo de ello). Los trabajos de Gino Germani (1965) y Ruth Sautu (1965) se preguntaron por el vínculo entre el origen social de los estudiantes de la UBA y la regularidad en sus estudios. Germani señaló el origen relativamente diverso de los estudiantes aunque remarcó el peso de los estratos medios y altos; por su parte, Sautu indagó, junto a los factores objetivos, aquellos de índole subjetiva que impactaban en el rendimiento de los estudiantes. Algunos años más tarde, Doris Klubischko (1980) analizó los censos universitarios de las décadas de 1950 a 1970 buscando demostrar que el aumento en la cantidad de estudiantes no había implicado necesariamente una democratización en el acceso de los sectores populares a la universidad.

A partir de la década de 1990, diversos autores examinaron las características de la universidad argentina en la posdictadura, destacando los alcances y problemas del nuevo y más amplio proceso de masificación universitaria iniciado en 1983. El pionero artículo de Bertoni y Cano (1990) esbozaba hace ya casi tres décadas la tensión existente entre el acelerado crecimiento de la matrícula durante los ochenta y un presupuesto estatal insuficiente para sostener esa estructura expandida, lo que se manifestaba en déficits de infraestructura y caída de los salarios. Desde la década del 2000, se publicaron diversos estudios que realizaron un balance y un diagnóstico de la universidad pública argentina en democracia (Chiroleu, 2005; Fernández Lamarra, 2002; Suasnábar, 2013; Mollis, 2001; Pérez
Lindo, 2017), entre los que se destaca el libro de Buchbinder y Marquina (2008), posiblemente uno de los más completos al trazar un recorrido histórico de 25 años del sistema universitario argentino.

Toda esta literatura reciente contiene a nuestro entender dos limitaciones en relación con el tema que buscamos desarrollar en este artículo. Por un lado, la pregunta específica sobre la condición de los estudiantes, si bien está presente en varios de ellos, carece del énfasis necesario para un análisis en profundidad sobre dicho aspecto. A la vez, se trata de trabajos sobre el conjunto del sistema universitario argentino, existiendo hasta la fecha muy limitadas investigaciones concretas sobre el desarrollo institucional de la UBA en democracia. Este hecho llama la atención en función de la magnitud de la universidad porteña y de su relevancia a escala nacional.

En relación con ambas cuestiones cabe destacar la labor de dos autores que se interrogaron sobre la realidad de la UBA desde el punto de vista de los estudiantes. Los trabajos de Mario Toer (1990 y 1998), apoyados en una serie de extensas y detalladas encuestas a centenares de alumnos, exponen una cantidad significativa de elementos que definen el perfil socioeconómico y cultural de los estudiantes en dos momentos determinados: la segunda mitad de la década de 1980 y la mitad de los años noventa. Por su parte, el texto de Sandra Carli (2012) indaga diferentes aspectos de la vida universitaria entre finales de los noventa y comienzos de los 2000 a partir de la experiencia estudiantil, tomando como base las narrativas de varios estudiantes entrevistados. De este modo, el texto da cuenta de un complejo entramado de vínculos, prácticas y representaciones en momentos de crisis de la universidad y el país. Estos dos trabajos sirven de base para el desarrollo de este artículo, en el que buscamos aportar una mirada más de largo plazo de los primeros 20 años de la UBA en democracia a partir del análisis de las características sociodemográficas de sus estudiantes. 


\section{La masificación de la UBA en los ochenta y noventa. Antecedentes y etapas}

Como referimos anteriormente, puede afirmarse que ya desde los años cincuenta la Universidad de Buenos Aires era una universidad masiva. Esta expansión fue parte de un proceso internacional, que se expresó con mayor intensidad en Argentina a partir de las características de la universidad desde la Reforma de 1918 y de las medidas que estableció el peronismo, como la eliminación de aranceles y la apertura del ingreso. En 1958, la UBA contaba con 58684 alumnos según el censo de ese año y tuvo un crecimiento de la matrícula del 61\% entre 1958 y 1972. ${ }^{5}$ Como señala Valeria Manzano (2017: 94), a principios de los años sesenta Argentina ocupaba el tercer puesto mundial en porcentaje de población matriculada en la universidad, con 756 estudiantes universitarios por cada 100000 habitantes. Los estudiantes universitarios pasaron del $5 \%$ de la población de edad de 20 a 24 años en 1950, al 11\% en 1960 y el 20\% en 1972 (Manzano, 2017: 95).

Cabe señalar que la política limitacionista de la dictadura de la autodenominada "Revolución Argentina”, a partir de 1966, forzó a una leve reducción de la matrícula en sus primeros años, pero importantes movilizaciones estudiantiles enfrentaron esa política y forzaron a una flexibilización de cupos y restricciones al ingreso. Como señalan Califa y Seia (2017), el movimiento estudiantil operó como un factor decisivo con relación al incremento de la matrícula en esos años, que desembocó en un nuevo e intenso salto en el número de ingresantes hacia 1973 y 1974.

El golpe de Estado de 1976, que inauguró la última y más sangrienta dictadura en Argentina, supuso un corte abrupto en este proceso, instaurando medidas elitistas y restrictivas en la universidad como cupos, exámenes de ingreso y aranceles, a la par de una dura represión al movimiento estudiantil. El objetivo declarado de esta política de "redimensionamiento", era achicar drásticamente la matrícula y "resolver" de este modo el problema político que suponía una universidad pública masiva (Seia, 2018). Los años finales de la última dictadura encontraron a un movimiento estudiantil nuevamente movilizado por el ingreso irrestricto y contra el gobierno de facto (Cristal, 2017). De este modo, la problemática del ingreso y la cuestión de la masividad de la universidad, en particular de la UBA, ya venían atravesando la agenda universitaria argentina desde los años cincuenta. Su centralidad en el periodo abierto en 1983, al que nos abocamos en este artículo, no radica tanto en la novedad del asunto sino en la inédita magnitud que alcanzó la matrícula universitaria a partir de entonces.

Efectivamente, como señalamos en la introducción, la cantidad de estudiantes de la UBA entre 1983 y 2004 prácticamente se triplicó. Un análisis pormenorizado de este incremento refleja que el mismo no fue continuo sino que existieron dos momentos de expansión acelerada en el periodo. El primero ocurrió en los cinco años posteriores a la salida de la última dictadura, y el segundo a fines de los noventa y principios de los 2000. En el lustro de 1983 a 1988, el número de alumnos creció un $69 \%$, mientras que en los ocho años que van de 1996 a 2004, aumentó un $60 \%$. En el tiempo intermedio entre ambos, la matrícula permaneció estable.

La primera etapa de la expansión se vinculó con la apertura democrática y la eliminación de las restricciones que había impuesto la dictadura. El gobierno de Raúl Alfonsín suprimió los cupos y aranceles y tras mantener un año el polémico examen de ingreso, fuertemente resistido por los estudiantes, en 1985 terminó instaurando en la UBA el Ciclo Básico Común $(\mathrm{CBC})$, forma de ingreso aún vigente. $\mathrm{El} \mathrm{CBC}$

\footnotetext{
${ }^{5}$ Series Estadísticas $\mathcal{N}^{\circ} 5$, publicadas por el Rectorado de la Universidad de Buenos Aires. Disponibles en: <http://www.uba.ar/institu-
} cional/censos/series/default.htm>. 
es un ciclo de un año de duración estimada, con seis materias que varían según la carrera elegida, al que los estudiantes acceden sin restricciones. Se cursa en sedes separadas de las facultades y debe ser aprobado en su totalidad para ingresar a las mismas. En este contexto, decenas de miles de estudiantes ingresaron a las aulas de la UBA entre 1983 y 1987, proceso paralelo al del conjunto del sistema universitario argentino, cuya población total pasó de 416000 estudiantes universitarios en 1983 a más de 700000 en 1986 (Buchbinder y Marquina, 2008: 27). Esta masificación no fue acompañada por un incremento presupuestario acorde, dando lugar a crecientes conflictos. Como señala Buchbinder (2005: 218), "el costo del crecimiento de la matrícula $[\ldots]$ fue compensado, principalmente, por los docentes y empleados administrativos, cuyos salarios disminuyeron de manera constante". Tras este primer salto en la matrícula, la misma permaneció estable hasta mediados de los noventa.

Tabla 1. Matrícula total

UBA

\begin{tabular}{|c|c|}
\hline Año & Matrícula total \\
\hline 1983 & 106793 \\
\hline 1988 & 180805 \\
\hline 1992 & 168808 \\
\hline 1996 & 183347 \\
\hline 2000 & 253260 \\
\hline 2004 & 293358 \\
\hline
\end{tabular}

Fuentes: elaboración propia con base en los censos de la UBA 1988, 1992, 1996, 2000 y 2004. Para 1983, tomamos el dato de la tesis doctoral de Guadalupe Seia (2019), quien se basó en datos brindados por el Departamento de Información Universitaria, sección de Políticas Universitarias, Ministerio de Educación de la Nación.

El segundo momento de expansión ocurrió entre finales de los noventa e inicios de los 2000, durante lo que Sandra Carli (2012: 17) denomina el "periodo de crisis", en relación con la profunda crisis económica y social que vivió Argentina en esos años y también a la crisis de la UBA como institución a partir de los sucesivos intentos de ajuste. En ese periodo la falta de opciones laborales empujó a una gran cantidad de jóvenes de estratos medios y medio-bajos a cursar la universidad como posible forma de progreso. También, a partir de la crisis generalizada, una parte de estudiantes con familias de ingresos medio-altos se volcó a la universidad pública en virtud de su gratuidad. Análogamente, entre 1996 y 2003 el conjunto de universidades nacionales incrementó su población total de 812308 estudiantes a 1251444 , un crecimiento del $54 \%{ }^{6}$

\footnotetext{
${ }^{6}$ Anuario 1996 de Estadísticas Universitarias, Programa de Mejoramiento del Sistema de Información Universitaria, Secretaría de Políticas Universitarias, Ministerio de Educación y Cultura de la Nación; Anuario 1999-2003 de Estadísticas Universitarias, Coordinación de Investigaciones e Información Estadística, Secretaría de Políticas Universitarias, Ministerio de Educación, Ciencia y Tecnología de la Nación.
} 
En suma, la apertura democrática primero y el "periodo de crisis" luego, implicaron dos momentos casi sucesivos de fuerte expansión de la matrícula de la Universidad de Buenos Aires, llegando a cifras totales cercanas a las que se mantienen hasta la actualidad. ${ }^{7}$ Como señalamos, el presupuesto estatal no acompañó este acelerado crecimiento. A continuación analizaremos las características y transformaciones dentro del estudiantado en todo este contexto.

\section{Características generales de los estudiantes. Edad, sexo, estado civil}

Un recorrido por algunas de las características demográficas del estudiantado de la UBA en estos años pone de manifiesto rasgos generales de su morfología. La edad promedio de los estudiantes, por ejemplo, se mantuvo relativamente estable a lo largo de todo el periodo, con alrededor de dos tercios de los alumnos en el rango entre 18 y 24 años y un 85\% menores a 30 años (ver tabla 2). La población estudiantil fue sin dudas una población joven, aun cuando fue relativamente significativa la proporción de alumnos con edades algo mayores. Este dato cobraba particular relieve en la década de los ochenta, en la medida en que el tercio de estudiantes mayores a 25 años portaba una experiencia distinta a los más nuevos: habían transitado toda o parte de la dictadura militar, e incluso si varios de ellos no habían vivido los años más crudos del terrorismo de Estado concentrados entre 1976 y 1979, muchos de ellos sí habían sufrido el clima de censura y las restricciones al ingreso que se mantuvieron hasta la salida del gobierno de facto. De este modo, la convivencia en los claustros entre camadas de estudiantes operó como nexo entre realidades políticas e institucionales disímiles y de algún modo contribuyó a la pervivencia de una memoria de lo que había sucedido durante la dictadura.

Tabla 2. Estudiantes de la UBA por edad

\begin{tabular}{|c|c|c|c|}
\hline Año & $\mathbf{1 8}$ a 24 años & $\mathbf{2 5}$ a 29 años & 30 años o más \\
\hline 1988 & $66 \%$ & $19 \%$ & $15 \%$ \\
\hline 1992 & $63 \%$ & $22 \%$ & $15 \%$ \\
\hline 1996 & $67 \%$ & $19 \%$ & $14 \%$ \\
\hline 2000 & $68 \%$ & $19 \%$ & $13 \%$ \\
\hline 2004 & $62 \%$ & $23 \%$ & $15 \%$ \\
\hline
\end{tabular}

Fuente: elaboración propia con base en censos de la UBA 1988, 1992, 1996, 2000 y 2004.

\footnotetext{
${ }^{7}$ Cabe señalar que en estos años se crearon nuevas carreras como Diseño Gráfico, Comunicación Social y Administración de Empresas que absorbieron a una parte de las nuevas camadas de estudiantes y que modificaron hasta cierto punto la distribución interna de esta población ampliada. No obstante, las disciplinas "profesionalistas" siguieron siendo entre 1985 y 2000 las más elegidas por los estudiantes, encabezadas por Abogacía, Medicina, Contador Público y Psicología, continuando la impronta ligada a la formación de profesionales liberales que signó a la UBA a lo largo de su historia (Buchbinder, 2005).
} 
Por otra parte, la cantidad de estudiantes mayores a 30 años variaba fuertemente según la facultad. En Filosofía y Letras alcanzó nada menos que el 26\% y el $31 \%$ en 1988 y 2000, frente a sólo el $13 \%$ y $17 \%$ en Medicina o el 8\% y 10\% en Arquitectura y Diseño en los mismos años. ${ }^{8}$ El tipo de carrera definió entonces un perfil de edad relativamente distinto según cada unidad académica, aunque predominara en todas el rango de 18 a 24 años.

En relación con el sexo, encontramos una creciente participación de las mujeres dentro de la matrícula estudiantil de la UBA. Si bien desde la década de los cincuenta la proporción de mujeres estudiantes había crecido sostenidamente (Manzano, 2017: 95), y en los setenta ya era casi igualitaria, el periodo que se abrió en 1983 fue el primero en la historia de la universidad porteña en el que su población femenina superó a la masculina, rasgo que se fue acentuando hasta la actualidad. En 2004, las estudiantes mujeres representaban el $61 \%$ del total de alumnos. En este aspecto también hay matices en la evolución de las distintas facultades. En 1988, todavía era mayoritaria la población

Tabla 3. Estudiantes de la UBA por sexo

\begin{tabular}{|c|c|c|}
\hline Año & Masculino & Femenino \\
\hline 1988 & $49 \%$ & $51 \%$ \\
\hline 1992 & $45 \%$ & $55 \%$ \\
\hline 1996 & $42 \%$ & $58 \%$ \\
\hline 2000 & $40 \%$ & $60 \%$ \\
\hline 2004 & $39 \%$ & $61 \%$ \\
\hline
\end{tabular}

Fuente: elaboración propia con base en censos de la UBA 1988, 1992, 1996, 2000 y 2004. masculina en seis de las 13 unidades académicas, mientras en 2004 sólo Agronomía e Ingeniería albergaban mayor cantidad de hombres que mujeres. El 82\% de varones en esta última facultad ese año contrastaba con el 83\% de mujeres que cursaban en Psicología. ${ }^{9}$

Por último, una mirada sobre el estado civil de los estudiantes refleja también permanencias a lo largo de los años examinados. La inmensa mayoría de los estudiantes declararon ser solteros y no tener hijos. Este dato da cuenta de que la mayor parte de los estudiantes no cargó con la obligación de sostener una familia propia, hecho que distingue a este sector de otros dentro de la juventud argentina. ${ }^{10}$ No obstante, en términos absolutos el número de estudiantes con hijos no fue menor, alcanzando casi 30000 personas en 2004.

Hasta aquí encontramos ciertas regularidades, con una amplia mayoría de estudiantes jóvenes, solteros, sin hijos y con creciente participación femenina. No obstante, el examen de los elementos socioeconómicos que desarrollamos a continuación pondrá de manifiesto una fuerte diversidad dentro de la población estudiantil en el periodo.

\section{Tabla 4. Estudiantes de la UBA por estado civil e hijos}

\begin{tabular}{|c|c|c|c|}
\hline Año & Soltero & Casado & Con hijos \\
\hline 1988 & $82 \%$ & $16 \%$ & $11 \%$ \\
\hline 2004 & $85 \%$ & $13 \%$ & $10 \%$ \\
\hline
\end{tabular}

Fuente: elaboración propia con base en censos de la UBA de 1988 y 2004

${ }^{8}$ Censos de la Universidad de Buenos Aires, 1988 y 2004.

${ }^{9}$ Censos de la Universidad de Buenos Aires, 1988 y 2004. El amplio predominio de la población estudiantil femenina en el periodo choca con la baja representación de las mujeres tanto en los organismos gremiales estudiantiles como a nivel de las autoridades universitarias. A modo de ejemplo, los 13 presidentes de centros de estudiantes electos en 1983 a la salida de la dictadura, así como el conjunto de los decanos normalizadores de las facultades nombrados en ese mismo lapso fueron hombres en su totalidad. Esta realidad no se modificó sustantivamente en las décadas subsiguientes, a pesar de la creciente y nítida mayoría femenina de la UBA. Todos los rectores de la Universidad desde 1983 a la actualidad, así como la inmensa mayoría de los presidentes de la Federación Universitaria de Buenos Aires (FUBA), fueron varones. De este modo, la UBA se transformó en los años de democracia en una universidad predominantemente femenina pero todavía con un sesgo fuertemente machista en relación con la representación institucional y gremial.

${ }^{10}$ Mario Toer (1990: 16) señala asimismo que el 77\% de los estudiantes vivía con sus padres en 1986. 


\section{Condiciones socioeconómicas. Residencia, trabajo, familia}

El análisis de factores tales como el lugar de residencia, la escuela secundaria, el trabajo propio y familiar, y el nivel de instrucción de los padres de los estudiantes de la UBA durante los 20 años en cuestión, da cuenta de una gran heterogeneidad de situaciones. El mapa se complejiza y muestra que en la universidad pública en democracia convivieron estudiantes con situaciones muy variadas.

Comenzando por el lugar de residencia, de un lado la inmensa mayoría de los estudiantes declaraba no haber cambiado de vivienda por motivo de los estudios, si bien el porcentaje se incrementó ligeramente entre 1988 y 2004. Este dato parece expresar que fueron relativamente pocos los estudiantes de la UBA que provinieron del interior del país o de países

Tabla 5. Estudiantes UBA por cambio de lugar de residencia a causa de sus estudios

\begin{tabular}{|c|c|c|}
\hline Año & Cambió & No cambió \\
\hline 1988 & $9 \%$ & $91 \%$ \\
\hline 2004 & $14 \%$ & $85 \%$ \\
\hline
\end{tabular}

Fuente: elaboración propia con base en censos de la UBA de 1988 y 2004.

En la medida en que todas las facultades y varias de las sedes del CBC se encontraban en la CABA, estos porcentajes nos hablan de una disparidad en relación con los traslados semanales de los estudiantes. Mientras una parte recorría distancias muy amplias, otros se encontraban relativamente cerca de sus lugares de estudio. La recurrente asociación entre Conurbano y sectores populares debe tomarse con cautela, ya que en muchas localidades viven sectores medios y medio-altos, aunque en términos generales el incremento del porcentaje de estudiantes viviendo en el Gran Buenos Aires podría pensarse limítrofes, si bien nuevamente eran un número considerable en términos absolutos. Sin duda, la gran mayoría de los estudiantes formó parte de familias asentadas en el territorio del Área Metropolitana de Buenos Aires.

Sin embargo, existió una gran paridad entre los estudiantes que vivían dentro de la Capital Federal (su denominación actual es Ciudad Autónoma de Buenos Aires, CABA) y aquellos que residían en los distintos partidos del Gran Buenos Aires (distribuidos de forma relativamente pareja entre sus zonas norte, oeste y sur). De hecho, el porcentaje de estudiantes que habitaban en el Conurbano bonaerense se incrementó en estas décadas, llegando casi a una paridad entre ambas (una parte muy pequeña que no consignamos residía en el interior de la provincia de Buenos Aires).

Tabla 6. Lugar de residencia de los estudiantes de la UBA

\begin{tabular}{|c|c|c|}
\hline Año & CABA & Gran Buenos Aires \\
\hline 1988 & $59 \%$ & $39 \%$ \\
\hline 2004 & $53 \%$ & $45 \%$ \\
\hline
\end{tabular}

Fuente: elaboración propia con base en censos de la UBA de 1988 y 2004.

como un indicador de cierta apertura de la UBA hacia sectores de extracción popular.

Los escenarios también variaban por facultad. Mientras en Filosofía y Letras los censos del 1988 y 2004 arrojaban un 68\% y 65\% de estudiantes viviendo en la CABA respectivamente, los estudiantes de Medicina lo hacían en un 58\% y 49\%. Es decir que más de la mitad de los estudiantes de esa importante facultad ubicada en una zona céntrica de la ciudad recorrían en 2004 distancias significativas desde el Conurbano para asistir a clase. Lo mismo puede pensarse en relación con Arquitectura, ubicada en 
la Ciudad Universitaria en el extremo norte de la CABA. Un 22\% en 1988 y 29\% en 2004 de sus estudiantes vivían en las alejadas zona oeste y sur del Gran Buenos Aires. Por su parte, en el conjunto del CBC el 56\% vivía en 2004 en las afueras de la ciudad, dato que retomaremos más adelante. ${ }^{11}$

La situación laboral de los estudiantes también muestra variaciones significativas. Por un lado, es llamativa la gran cantidad de estudiantes que trabajan, entre el 58 y el 72\% en los 20 años considerados (ver tabla 7), si bien su número ya era alto en las décadas de 1950 y 1960 (Germani, 1965). Entre el 40 y el 50\% del total, trabajaba más de 25 horas por semana, es decir con una dedicación diaria sustantiva (ver tabla 7). Según las encuestas de Mario Toer para finales de los ochenta, la mayor proporción de trabajadores se ubicaba en niveles socioeconómicos más bajos $(\mathrm{C} 2$ y D) con un 65\% y 71\%, aunque "el porcentaje de [estudiantes en el estrato] AB que trabaja (43\%) es por demás significativo y contrasta con los índices habituales de otros países", lo que daría cuenta de que "se ha configurado una valoración cultural [sobre el trabajo] que incluye a quienes hipotéticamente podrían prescindir de hacerlo" (Toer, 1990: 43).

El aumento del porcentaje de estudiantes que no trabajaban a fines de los noventa se relaciona centralmente con las consecuencias de la crisis en Argentina, en momentos en que el desempleo escaló dramáticamente y más aún el desempleo juvenil. En este sentido, en 1996 y 2000 más del 60\% de los estudiantes que no trabajaban declaraba estar buscando trabajo. $^{12}$

Tabla 7. Situación laboral de los estudiantes de la UBA

\begin{tabular}{|c|c|c|c|}
\hline Año & No trabaja & $\begin{array}{c}\text { Trabaja hasta 25h } \\
\text { por semana }\end{array}$ & $\begin{array}{c}\text { Trabaja más de } \\
\text { 25h por semana }\end{array}$ \\
\hline 1988 & $33 \%$ & $19 \%$ & $48 \%$ \\
\hline 1992 & $28 \%$ & $22 \%$ & $50 \%$ \\
\hline 1996 & $41 \%$ & $19 \%$ & $40 \%$ \\
\hline 2000 & $42 \%$ & $17 \%$ & $41 \%$ \\
\hline 2004 & $42 \%$ & $13 \%$ & $45 \%$ \\
\hline
\end{tabular}

Fuente: elaboración propia con base en censos de la UBA 1988, 1992, 1996, 2000 y 2004.

Las diferencias entre facultades eran notorias en este terreno. Mientras en la Facultad de Medicina no trabajaban el 50\% y el 60\% en 1988 y 2004, en Ciencias Económicas los estudiantes con empleo constituían el $83 \%$ y el $74 \%$ respectivamente, con un alto porcentaje ocupado más de 25 horas. En el CBC, la mayoría de los estudiantes trabajaban en todos los años relevados. Asimismo, es importante contemplar las divergencias dentro del propio universo de los estudiantes que trabajaban. Los testimonios recogidos por Sandra Carli (2012), muestran las profundas diferencias entre estudiantes que trabajaban unas horas y otros que lo hacían durante todo el día. Toer (1990: 22) proponía en este sentido una diferenciación interesante entre "estudiantes que trabajan" y "trabajadores que estudian". Las categorías son desde ya analíticas y la frontera entre ambos grupos es porosa pero resulta útil en el sentido de observar

\footnotetext{
${ }^{11}$ Censos de la Universidad de Buenos Aires, 1988 y 2004.

${ }^{12}$ Censos de la Universidad de Buenos Aires, 1996 y 2000.
} 
la diversidad de situaciones laborales entre los estudiantes. De este modo, el autor planteaba que para fines de los ochenta "con cautela podríamos concluir entonces que los trabajadores que estudian se encuentran entre la cuarta parte y el tercio del total de estudiantes" (Toer, 1990: 22). Según el autor también se verificaban diferencias según lo avanzado de la carrera, con un aumento del $5 \%$ de los estudiantes que trabajaban entre quienes tenían más de diez materias de sus carreras aprobadas. A la vez, en 1988 el trabajo masculino superaba al femenino en nada menos que 15 puntos. Es decir que junto al alto porcentaje de estudiantes que trabajan, la multiplicidad de situaciones era remarcable.

Lo mismo puede señalarse en relación con el peso de los ingresos del propio trabajo en el sostenimiento personal. La fuente principal de ingresos de los estudiantes se repartía de forma relativamente equitativa entre el trabajo propio y el aporte de los padres o familiares. Nuevamente aquí el periodo de crisis empujó a un incremento en el aporte de las familias al sostenimiento de los estudiantes. Menos del 1\% declaraba sostenerse a través de becas, lo que evidencia el fuerte déficit presupuestario en este plano.

Por último, cabe ponderar el tipo de trabajo de los estudiantes. Predominaban los trabajadores administrativos y empleados calificados (entre $40 \mathrm{y}$ $48 \%$ del total de estudiantes en 1992, 1996 y 2000), aunque también existía un porcentaje menor de trabajadores manuales, con o sin calificación, que representaban entre el 6 y el $7 \%$, porcentajes similares a los que se desempeñaron como jefes intermedios, profesionales, gerentes, dueños de PyMES o altos jefes. El porcentaje de trabajadores manuales saltaba al $10 \%$ entre los que cursaban el CBC y se reducía al 2-4\% en facultades como Ciencias Económicas. ${ }^{13}$

Tabla 8. Fuente principal de ingresos de los estudiantes de la UBA

\begin{tabular}{|c|c|c|}
\hline Año & Trabajo propio & $\begin{array}{c}\text { Aporte de padres o } \\
\text { familiares }\end{array}$ \\
\hline 1988 & $58 \%$ & $41 \%$ \\
\hline 2004 & $48 \%$ & $49 \%$ \\
\hline
\end{tabular}

Fuente: elaboración propia con base en censos de la UBA de 1988 y 2004.

Para completar el cuadro en relación con las condiciones socioeconómicas de los estudiantes de la UBA cabe indagar la situación de sus familias, tanto en relación con el trabajo como con los niveles de instrucción de los padres. En relación con el aspecto laboral, tomaremos como dato de referencia el trabajo del padre, en la medida en que en la mayoría de los casos el hombre siguió siendo el sostén de hogar. Como muestra la tabla 9, la distribución entre diferentes categorías refleja orígenes bastante diversos entre los estudiantes. Los padres en trabajos administrativos como de empleados calificados abarcaban entre el 26 y $30 \%$ según el año, siendo ésta la categoría más numerosa. Pero con proporciones cercanas se ubican de un lado los trabajadores manuales (con o sin calificación) que rondaban un notorio $20 \%$ y del otro los jefes intermedios o profesionales universitarios también en torno al 20\%. Pocos puntos por debajo encontramos a gerentes, directores y dueños de PyMES a los que se suma un porcentaje menor de altos jefes.

\footnotetext{
${ }^{13}$ Censos de la Universidad de Buenos Aires, 1992, 1996 y 2000.
} 
Tabla 9. Ocupación del padre de los estudiantes de la UBA

\begin{tabular}{|c|c|c|c|c|c|}
\hline Año & $\begin{array}{c}\text { Tr. Manual } \\
\text { (con o sin calif.) }\end{array}$ & $\begin{array}{c}\text { Tr. Administrativo / } \\
\text { Empleado calificado }\end{array}$ & $\begin{array}{c}\text { Jefe intermedio / Prof. } \\
\text { universit. }\end{array}$ & $\begin{array}{c}\text { Gerente / Dir. / Dueño } \\
\text { / Socio (PyMEs) }\end{array}$ & $\begin{array}{c}\text { Gerente / Dir. / Dueño } \\
\text { / Socio / Altos jefes }\end{array}$ \\
\hline 1992 & $19 \%$ & $30 \%$ & $23 \%$ & $17 \%$ & $4 \%$ \\
\hline 1996 & $19 \%$ & $28 \%$ & $20 \%$ & $14 \%$ & $3 \%$ \\
\hline 2000 & $21 \%$ & $26 \%$ & $19 \%$ & $18 \%$ & \\
\hline
\end{tabular}

Fuente: elaboración propia con base en censos de la UBA 1992, 1996, 2000. No se incluyeron los censos 1988 y 2004 ya que esta parte fue elaborada con criterios no comparables. Tampoco incluimos las categorías "Otros" y "No responde". El Censo 2000 no distingue entre gerentes y dueños de pymes y altos jefes de empresas.

Como se observa, los porcentajes que representan cada una de las cuatro categorías ocupacionales descritas son relativamente equitativos entre sí, dando cuenta de cierta heterogeneidad en los orígenes familiares del estudiantado, más allá de que los sectores medios tendieron a concentrar una proporción central. En un sentido análogo, al estudiar los niveles de ingreso de los padres de los alumnos a fines de los ochenta (que no necesariamente coincidían con los rubros ocupacionales), Toer (1990: 22) halló un 14\% en la categoría más alta $\mathrm{AB}$, un $36 \%$ en $\mathrm{C} 1$, un $36 \%$ en C2 y un $15 \%$ en D, lo que según el autor daría como resultado "una curva de Gauss casi perfecta". Por otra parte, en este terreno también deben contemplarse las diferencias entre las unidades académicas. En el CBC, un 22\% de los estudiantes tenían padres que se desempeñaban como trabajadores manuales en 1992, cifra que escaló al 26\% en 2000, mientras en Ingeniería en esos mismos años los porcentajes alcanzaban sólo el 12 y el $14 \%{ }^{14}$

Otro indicador del origen social de los estudiantes es el tipo de escuela secundaria al que asistieron. Como se observa en la tabla 10, la distancia entre pública y privada es reducida y llama la atención el incremento paulatino de la privada, que supera a la pública desde fines de los noventa. Este dato podría vincularse con el deterioro general de la escuela pública y el crecimiento más general de las instituciones privadas de enseñanza media en democracia. También con el hecho de que a fines de los noventa, la crisis "forzó a un mayor acercamiento a la UBA —al menos transitorio - de jóvenes de sectores medios y medio-altos procedentes de escuelas privadas, como resultado de las debacles familiares" (Carli, 2012: 80).

Tabla 10. Tipo de escuela secundaria al que asistieron los estudiantes de la UBA

\begin{tabular}{|c|c|c|}
\hline Año & Pública & Privada \\
\hline 1988 & $57 \%$ & $42 \%$ \\
\hline 1992 & $54 \%$ & $45 \%$ \\
\hline 1996 & $49 \%$ & $49 \%$ \\
\hline 2000 & $46 \%$ & $53 \%$ \\
\hline 2004 & $43 \%$ & $55 \%$ \\
\hline
\end{tabular}

Fuente: elaboración propia con base en censos de la UBA 1988, 1992, 1996, 2000 y 2004.

\footnotetext{
${ }^{14}$ Censos de la Universidad de Buenos Aires, 1992 y 2000.
} 
La investigación de Toer (1990: 33) agrega un matiz estadístico al discriminar dentro de los secundarios privados a los colegios religiosos. La población que en 1988 había asistido a ese tipo de establecimientos ascendía al 31\% e incluso un 36\% dentro de la población femenina. Se trata de un dato llamativo para una universidad con una fuerte tradición laica. Si bien el porcentaje era mayor en el estrato de ingresos $\mathrm{AB}(37 \%)$, era remarcable también en el estrato D con un $22 \%$.

Finalmente, el nivel de instrucción de los padres es otro factor relevante para dar cuenta de los orígenes familiares, así como para complejizar la composición de los "sectores medios" a los que nos referimos anteriormente. ${ }^{15}$ En primer lugar, llama la atención que más de la mitad de los estudiantes fueron primera generación de universitarios no sólo en 1988 (dónde constituían más del 60\%) sino incluso en 2004 (ver tabla 11). Este dato reafirma el papel de la universidad como espacio que concentró las aspiraciones de progreso social de una parte de la población (aunque como veremos en el próximo apartado, la realidad universitaria posterior que vivieron los estudiantes haya sido bastante más compleja).

Tabla 11. Nivel de instrucción de los padres de los estudiantes de la UBA

\begin{tabular}{|c|c|c|c|c|c|c|c|c|c|c|}
\hline & \multicolumn{5}{|c|}{ Nivel de instrucción del padre } & \multicolumn{5}{|c|}{ Nivel de instrucción de la madre } \\
\hline Año & 1988 & 1992 & 1996 & 2000 & 2004 & 1988 & 1992 & 1996 & 2000 & 2004 \\
\hline Primario comp. o inc. & $31 \%$ & $27 \%$ & $23 \%$ & $22 \%$ & $20 \%$ & $37 \%$ & $31 \%$ & $25 \%$ & $22 \%$ & $20 \%$ \\
\hline Secundario comp. o inc. & $34 \%$ & $33 \%$ & $33 \%$ & $34 \%$ & $33 \%$ & $38 \%$ & $36 \%$ & $35 \%$ & $36 \%$ & $34 \%$ \\
\hline Terciario no univ. comp. o inc. & $10 \%$ & $8 \%$ & $8 \%$ & $8 \%$ & $8 \%$ & $15 \%$ & $15 \%$ & $17 \%$ & $17 \%$ & $18 \%$ \\
\hline Universitario incompleto & $7 \%$ & $11 \%$ & $12 \%$ & $12 \%$ & $12 \%$ & $4 \%$ & $7 \%$ & $9 \%$ & $9 \%$ & $9 \%$ \\
\hline Universitario completo & $17 \%$ & $21 \%$ & $24 \%$ & $24 \%$ & $24 \%$ & $6 \%$ & $11 \%$ & $15 \%$ & $16 \%$ & $17 \%$ \\
\hline
\end{tabular}

Fuente: elaboración propia con base en censos de la UBA 1988, 1992, 1996, 2000 y 2004.

La heterogeneidad en este terreno vuelve a ser evidente. En 2004 convivían en la UBA entre 20 y 25\% de estudiantes cuyos padres o madres tenían título universitario con otro $20 \%$ con padres o madres sólo con estudios primarios, en algunos casos incompletos. Las diferencias entre unidades académicas y facultades también se acentuaban. En 2004, un 58\% de los estudiantes del CBC tenían padres con estudios primarios o secundarios, mientras en Ingeniería ese porcentaje era del 36\%. Al revés, un 19\% de los padres de alumnos del CBC tenían estudios universitarios contra un $41 \%$ de los de Ingeniería. Entre esos extremos aparecían casos como los de Medicina, donde en 2000 la proporción de padres con estudios primarios era de un significativo $24 \%$ o Arquitectura con un $30 \%$ de padres con título universitario. ${ }^{16}$

\footnotetext{
${ }^{15}$ Ya Doris Klubischko (1980: 64) había planteado que "sería un error tratar a los diferentes grupos que componen a los sectores medios como formando una unidad. En efecto, los datos revelan que dentro de este agregado se perfilan pautas muy disímiles. Más aún, esas diferencias se hicieron evidentes no tanto al introducir la clásica dicotomía entre sector medio dependiente e independiente, sino más bien al especificar los antecedentes culturales y más concretamente el nivel educativo".

${ }^{16}$ Censos de la Universidad de Buenos Aires 2000 y 2004.
} 
En suma, el análisis de los factores socioeconómicos relevados nos permite observar que la marcada diversidad de situaciones fue una de las características más relevantes dentro de la población estudiantil en democracia. Esta heterogeneidad nos habla de cierta expansión hacia sectores que accedían por primera vez a la universidad, pero también de una significativa fragmentación de los estudiantes, a partir de realidades bastante disímiles por facultad y año de cursada, que pudo haber condicionado sus formas de socialización así como sus posibilidades de acción colectiva. En este sentido, el CBC, primer año de las carreras que funcionó y sigue funcionando como una unidad académica separada, constituye un lugar a observar con detenimiento. Como pudimos registrar, el CBC concentró la mayor proporción de estudiantes con familias de estratos socioeconómicos bajos o medio-bajos, con padres con menores niveles de instrucción, con más estudiantes que trabajaban y que vivían en el Conurbano Bonaerense. En el próximo apartado buscamos vincular estos factores con los altos niveles de deserción que se registraron en las décadas investigadas.

\section{El СвC y las tasas de deserción estudiantil}

Desde su creación en 1985, el Ciclo Básico Común albergó a miles de estudiantes en sedes situadas por fuera de las facultades (o en áreas específicas en el caso de los edificios de Ciudad Universitaria). En el periodo investigado, los estudiantes del CBC representaron entre el 24 y el 30\% del total de alumnos de la UBA, una proporción central (ver tabla 12). En términos absolutos las cifras son aún más impactantes. En 2000, los estudiantes del CBC eran nada menos que 76 788, probablemente uno de los ámbitos de sociabilidad juvenil más importantes de Buenos Aires (ver tabla 12). Indudablemente, la ampliación de la matrícula desde el regreso de la democracia pasó por (y se concentró en gran medida en) el CBC. ¿Hasta qué punto logró ese Ciclo absorber y retener a esa masa de ingresantes?

Llamativamente, no contamos con datos estadísticos fehacientes en relación con las tasas de deserción de la UBA y del CBC en particular. Para estimarlas, hemos comparado el número de ingresantes a las facultades de cada año publicados por el Rectorado de la UBA con los ingresos al CBC del año inmediatamente anterior. Aunque el resultado es estimativo, en general las variaciones se anulan entre un año y otro y el promedio global se aproxima de forma bastante precisa a las tasas de deserción efectivas.

Tabla 12. Estudiantes del CBC. Total y porcentaje sobre estudiantes de la UBA

\begin{tabular}{|c|c|c|}
\hline Año & Total CBC & \% sobre total UBA \\
\hline 1988 & 43,932 & $24 \%$ \\
\hline 1992 & 46,345 & $27 \%$ \\
\hline 1996 & 51,480 & $28 \%$ \\
\hline 2000 & 76,788 & $30 \%$ \\
\hline 2004 & 78,681 & $27 \%$ \\
\hline
\end{tabular}

Fuente: elaboración propia con base en censos de la UBA 1988, 1992, 1996, 2000 y 2004. 
Tabla 13. Ingresantes a las carreras frente a ingresos al CBC del año anterior

\begin{tabular}{|c|c|c|c|c|c|c|c|c|c|}
\hline Año & 1986 & 1987 & 1988 & 1989 & 1990 & 1991 & 1992 & 1993 & 1994 \\
\hline Carreras & 33,119 & 28,901 & 23,655 & 18,714 & 18,743 & 17,891 & 19,732 & 21,717 & 23,924 \\
\hline$C B C$ año anterior & 65,760 & 61,926 & 43,707 & 39,852 & 37,592 & 36,612 & 42,471 & 40,394 & 39,871 \\
\hline$\%$ & $50 \%$ & $47 \%$ & $54 \%$ & $47 \%$ & $50 \%$ & $49 \%$ & $46 \%$ & $54 \%$ & $60 \%$ \\
\hline
\end{tabular}

\begin{tabular}{|c|c|c|c|c|c|c|c|c|c|c|}
\hline Año & 1995 & 1996 & 1997 & 1998 & 1999 & 2000 & 2001 & 2002 & 2003 & 2004 \\
\hline Carreras & 25,493 & 30,698 & 28,053 & 32,837 & 33,319 & 32,687 & 31,694 & 34,247 & 36,473 & 36,946 \\
\hline CBC año anterior & 44,238 & 50,307 & 53,000 & 55,448 & 61,274 & 66,548 & 65,000 & 66,757 & 69,214 & 71,021 \\
\hline$\%$ & $58 \%$ & $61 \%$ & $53 \%$ & $59 \%$ & $54 \%$ & $49 \%$ & $49 \%$ & $51 \%$ & $53 \%$ & $52 \%$ \\
\hline
\end{tabular}

Fuente: elaboración propia con base en Series Estadísticas N5 publicadas por el Rectorado de la UBA.

El promedio general que resulta de esta tabla es una proporción de ingresos a las Facultades del $52 \%$ o, visto a la inversa, una deserción en el CBC del $48 \%$ anual. ${ }^{17}$ Es decir que entre 1986 y 2004, uno de cada dos estudiantes que ingresó al CBC no pudo terminarlo. En números absolutos, ingresaron al CBC entre 1985 y 2003, 1010992 estudiantes, y a las carreras, entre 1986 y 2004, 528843 alumnos. De este modo, en casi 20 años quedaron en el camino nada menos que 500000 estudiantes.

Como vimos en el apartado anterior, el CBC concentró estudiantes con condiciones socioeconómicas más vulnerables que el promedio de la UBA. Siguiendo los datos del censo 2004, allí era mayor la proporción de estudiantes con padres que se desempeñaban como trabajadores manuales ( $26 \%$ frente a $21 \%$ del promedio), con padres con niveles de instrucción primario o secundario (58\% frente a 53\% del promedio), y con lugar de residencia en el Gran Buenos Aires (56\% frente a $45 \%$ del promedio). Considerando que el propio CBC estaba incluido en los promedios generales, la distancia con el resto de la UBA era aún mayor.
Si comparamos estos datos con las tasas de deserción calculadas, se vuelve evidente que los sectores con condiciones socioeconómicas más difíciles, con menor nivel educativo en el ámbito familiar, con lugares de residencia más alejados, o con situaciones laborales más demandantes, son los que más abandonaron sus estudios en el primer año. Mario Toer (1998) llega a una conclusión similar: existe un vínculo directo entre nivel socioeconómico y nivel educativo de los padres con el rendimiento de los estudiantes.

Los inquietantes datos sobre la deserción en el CBC se complementan con aquellos que surgen de evaluar las tasas de graduación de la UBA. ¿Cuántos de los estudiantes que ingresan a la UBA logran recibirse? Nuevamente este dato no existe ni en los censos de la UBA ni en los anuarios de la Secretaría de Políticas Universitarias. Por ese motivo, realizamos un cálculo en términos análogos a la tabla anterior. Comparamos el número absoluto de graduados por año con el total de ingresantes al CBC seis años antes, tomando ese lapso como duración promedio estimada de las carreras de la UBA. Otra vez, el resultado es aproximado pero en

\footnotetext{
${ }^{17}$ Sandra Carli (2012: 61) estima datos similares o incluso superiores: "Los datos sobre deserción en el primer año, si bien no constan en los documentos analizados, rondan entre el 50 y el $60 \%$ ".
} 
Tabla 14. Graduados UBA frente a ingresos al CBC seis años antes

\begin{tabular}{|c|c|c|c|c|c|c|c|c|}
\hline Año & $\mathbf{1 9 9 1}$ & $\mathbf{1 9 9 2}$ & $\mathbf{1 9 9 3}$ & $\mathbf{1 9 9 4}$ & $\mathbf{1 9 9 5}$ & $\mathbf{1 9 9 6}$ & $\mathbf{1 9 9 7}$ \\
\hline Graduados & 12,050 & 12,194 & 15,809 & 13,434 & 11,062 & 10,983 & 11,162 \\
\hline Ingresos al CBC 6 años antes & 65,760 & 61,926 & 43,707 & 39,852 & 37,592 & 36,612 & 42,471 \\
\hline \% & $18 \%$ & $20 \%$ & $36 \%$ & $34 \%$ & $29 \%$ & $30 \%$ & $26 \%$ \\
\hline Año & 1998 & $\mathbf{1 9 9 9}$ & $\mathbf{2 0 0 0}$ & $\mathbf{2 0 0 1}$ & $\mathbf{2 0 0 2}$ & $\mathbf{2 0 0 3}$ & $\mathbf{2 0 0 4}$ \\
\hline Graduados & 11,130 & 11,388 & 13,991 & 13,159 & 14,187 & 15,063 & 15,647 \\
\hline Ingresos al CBC 6 años antes & 40,394 & 39,871 & 44,238 & 50,307 & 53,000 & 55,448 & 61,274 \\
\hline
\end{tabular}

Fuente: elaboración propia con base en Series Estadísticas N5 publicadas por el Rectorado de la UBA.

el mediano plazo tiende a dar cuenta de modo bastante fehaciente de la realidad.

En total, entre 1985 y 1998 ingresaron al CBC de la UBA 672452 estudiantes y entre 1991 y 2004 se graduaron 181 259. La relación entre ambos números es del $27 \%$, alrededor de 1 de cada 4 alumnos, o dicho de otro modo por cada 4 estudiantes que ingresan al CBC sólo uno se gradúa seis años después. $\mathrm{Al}$ revés, la tasa de deserción total de la UBA se ubicaría en el 73\%, cifra similar al 70\% que estima Toer (1998). Es decir que aproximadamente 3 de cada 4 estudiantes de la UBA no terminan sus estudios (y como vimos, 2 de esos 3 que abandonan lo hacen durante el primer año, en el CBC). ${ }^{18}$

Nuevamente aparece la variable socioeconómica en relación con estos guarismos. Como señala Carli (2012: 63), "mientras en la Argentina seis de cada diez jóvenes que ingresaban a la universidad procedían de sectores medios y altos, el 78\% de los que se graduaban pertenecían a esos sectores, lo cual indica el peso de la condición social en un contexto general de empobrecimiento en el que se dificulta la terminación de los estudios". En definitiva, las altas tasas de deserción en el CBC y en la UBA en general, y su reverso en las bajas tasas de graduación, ponen de manifiesto uno de los principales problemas de la universidad pública en estos años.

\section{Conclusiones}

Hemos examinado una serie de variables sobre la población estudiantil de la UBA entre 1983 y 2004 que dan cuenta de una serie de características y problemáticas de la universidad pública en democracia. En primer lugar existen ciertos patrones generales que se repiten en estos más de 20 años. La mayoría de los estudiantes de la UBA fueron jóvenes de entre 18 y 29 años, solteros, sin hijos y que procedían del área metropolitana de Buenos Aires. Todos estos rasgos englobaron a más del 85\% de la población estudiantil de la universidad porteña. Si bien el número de madres estudiantes, de alumnos mayores a 30 años o de alumnos provenientes del interior de Argentina no es desdeñable, sobre todo en términos absolutos, los rasgos antes enumerados fueron ampliamente predominantes.

\footnotetext{
${ }^{18}$ Ya en su estudio a mediados de los sesenta, Germani (1965: 9) alertaba que "Uno de los problemas más serios que debe enfrentar la Universidad en la Argentina es el de la elevada proporción de abandonos o de estudiantes que no cumplen con regularidad sus estudios, y que emplean — cuando los terminan - periodos muchos más largos que el previsto en los respectivos planes de estudio".
} 
A partir de estas características comunes básicas, se abría un abanico que expresaba una profunda heterogeneidad dentro del estudiantado, no sólo en relación con la carrera elegida o el sexo, sino en particular con su procedencia, trabajo y condiciones socioeconómicas. De este modo, elementos como la situación laboral propia, la escuela secundaria, el empleo de los padres o su nivel de instrucción reflejan una pluralidad de situaciones y grafican la "base de reclutamiento amplia" (Toer, 1998) que tuvo la UBA en democracia a partir de la apertura en el ingreso. En este sentido, fue significativa la proporción de estudiantes provenientes de estratos de ingreso bajos o medio-bajos, de hijos de trabajadores manuales, que trabajaban ellos mismos más de 25 horas por semana o que eran aun en 2004 primera generación de universitarios. Siguió predominando un origen ligado a sectores medios, pero dentro de los mismos existieron una enorme multiplicidad de afluentes, donde las diferencias internas en lo económico o en los niveles de formación de las familias fueron sustantivas y se fueron acentuando con las sucesivas crisis del país. En particular, aquella ocurrida a fines de los noventa y comienzos de los 2000 generó un empobrecimiento paulatino de la clase media del que la universidad no fue ajena. De este modo, en estos 20 años convivieron en la UBA estudiantes con una gama de situaciones muy amplia y compleja, y es indudable que la apertura en el ingreso fue una de las bases de esta diversidad. La heterogeneidad del estudiantado de la UBA en democracia constituye entonces una de sus características más relevantes.
Asimismo, hemos analizado cómo los sectores con condiciones socioeconómicas más vulnerables tendieron a abandonar más sus estudios, con una preocupante tasa de deserción en el CBC cercana al 50\%. En este sentido, como señaló Sandra Carli (2012: 64), "el ingreso irrestricto, al recibir jóvenes de distintos sectores sociales, tuvo un resultado contrario a sus aspiraciones: la mitad de los estudiantes no estuvieron en condiciones de permanecer y terminar los estudios". El ingreso irrestricto fue y sigue siendo una bandera fundamental del movimiento estudiantil de la UBA y la apertura del ingreso que se inició en los ochenta es reivindicada como una conquista. No obstante, el fuerte aumento de la matrícula de la UBA no fue acompañado por un aumento presupuestario acorde y el resultado, además de la precariedad laboral de docentes y trabajadores no-docentes, se hizo evidente en la incapacidad de la Universidad para retener a miles de estudiantes que todos los años ingresaron a sus claustros. La deserción estudiantil, particularmente la de jóvenes con condiciones socioeconómicas adversas, se transformó de este modo en uno de los problemas nodales de la UBA en democracia, y define una frontera entre las características más amplias entre el estudiantado del CBC y el de las facultades. En este sentido, cabe citar el trabajo de Doris Klubischko (1980: 75), quien hace ya casi 40 años advertía que "un proceso de democratización universitaria, expresado en una diversificación del espectro socioeconómico del estudiantado universitario, no es una consecuencia fácil, ni menos automática, de la expansión de la matrícula". 


\section{Referencias}

Bertoni, María de la Luz y Daniel Cano (1990), "La educación superior en la Argentina en los últimos veinte años: tendencias y políticas", Propuesta Educativa, núm. 2, pp. 11-24.

Buchbinder, Pablo (2005), Historia de las universidades Argentinas, Buenos Aires, Sudamericana.

Buchbinder, Pablo y Mónica Marquina (2008), Masividad, heterogeneidad y fragmentación: el sistema universitario argentino 1983-2008, Buenos Aires, UNGS/Biblioteca Nacional.

Califa, Juan Sebastián y Guadalupe Seia (2017), "La ampliación del sistema universitario argentino durante la 'Revolución Argentina'. Un estudio de sus causas a través de la Universidad de Buenos Aires (1969-1973)", A Contracorriente, vol. 15, núm. 1, pp. 36-59.

Carli, Sandra (2012), El estudiante universitario. Hacia una historia del presente de la educación pública, Buenos Aires, Siglo XXI.

Chiroleu, Adriana (2005), "La educación superior en la agenda de gobierno argentina en veinte años de democracia (1983-2003)", en Eduardo Rinesi, Germán Soprano y Claudio Suasnábar (comps.), Universidad, reformas y desafios: dilemas de la educación superior en la Argentina y el Brasil, Buenos Aires, UNGS/Prometeo, pp. 57-79.

Fernández Lamarra, Norberto (2002), La educación superior en la Argentina, Buenos Aires, OREALC/UNESCOEDUNTREF.

Ferreyra, María Marta, Ciro Avitabile, Javier Botero Álvarez, Francisco Haimovich Paz y Sergio Urzúa (2017), Momento decisivo: la educación superior en América Latina y el Caribe, Washington, DC, Banco Mundial.

Germani, Gino (1965), "El origen social de los estudiantes y la regularidad de los estudios", en Gino Germani y Ruth Sautu (comps.), Regularidad y origen social en los estudiantes universitarios, Buenos Aires, Publicaciones del Instituto de Sociología.
Klubischko, Doris (1980), El origen social de los estudiantes de la Universidad de Buenos Aires, Buenos Aires, Programa de las Naciones Unidas para el Desarrollo.

Manzano, Valeria (2017), La era de la juventud en la Argentina. Cultura, politica y sexualidad desde Perón hasta Videla, Buenos Aires, Fondo de Cultura Económica.

Mollis, Marcela (2001), La universidad argentina en tránsito, Buenos Aires, Fondo de Cultura Económica.

Pérez Lindo, Augusto (2017), La educación superior argentina (1983-2015). Diagnóstico y prospectiva, Buenos Aires, Eudeba.

Sautu, Ruth (1965), "Factores sociales de la regularidad en los estudios en la Universidad de Buenos Aires", en Gino Germani y Ruth Sautu (comps.), Regularidad y origen social en los estudiantes universitarios, Buenos Aires, Publicaciones del Instituto de Sociología.

Seia, Guadalupe (2019), "De la revolución a la reforma. Reconfiguraciones de las formas de militancia estudiantil en la Universidad de Buenos Aires entre 1976 y 1983", Tesis de Doctorado en Ciencias Sociales (no publicada), Buenos Aires, Facultad de Ciencias Sociales, Universidad de Buenos Aires.

Seia, Guadalupe (2018), "La aplicación de la política de "re-dimensionamiento" en la Universidad de Buenos Aires durante la última dictadura (1976-1983)", Revista Iberoamericana de Educación Superior (RIES), vol. IX, núm. 25.

Suasnábar, Claudio (2013), "Las políticas universitarias en 30 años de democracia: continuidades, rupturas y algunas lecciones de la experiencia", Cuestiones de sociología, núm. 9, <https://www.cuestionessociologia.fahce.unlp.edu.ar/ article/view/CSn09a45> [consulta: diciembre de 2019].

Toer, Mario (1998), El perfil de los estudiantes de la UBA: el trabajo, la política, la religión, los medios, Buenos Aires, Eudeba.

Toer, Mario (1990), Cómo son los estudiantes. Perfil socioeconómico y cultural de los estudiantes de la UBA, Buenos Aires, ECACatálogos.

\section{Cómo citar este artículo:}

Cristal, Yann (2021), "Masificación universitaria y población estudiantil. El estudiantado de la UBA en dos décadas de democracia (1983-2004)", Revista Iberoamericana de Educación Superior (RIES), vol. XII, núm. 33, pp. 101-118, DoI: https://doi.org/10.22201/ iisue.20072872e.2021.33.860 [Consulta: fecha de última consulta]. 